\title{
Análise da Noção de Variabilidade Comportamental ${ }^{1}$
}

\author{
Maria Helena Leite Hunziker ${ }^{2}$ \\ Universidade de São Paulo \\ Rafael Moreno \\ Universidad de Sevilla
}

\begin{abstract}
RESUMO - O presente trabalho faz uma análise do conceito de variabilidade comportamental com o objetivo de padronizar as diferentes definições e usos do termo. Propomos: 1) uma definição que recorra ao ponto comum dentre os diversos conceitos de variabilidade existentes na literatura, e 2) a descrição e sistematização dos aspectos específicos de cada uso do termo, utilizando os seguintes critérios: conteúdo e estrutura de cada conceito, níveis de análise empírico/teórico, molar/ molecular, e tipos de medida e códigos com que são especificados. Essa análise é uma tentativa inicial para a construção de uma taxonomia da variabilidade comportamental.
\end{abstract}

Palavras-chave: variabilidade comportamental; seleção do comportamento; aprendizagem; análise metodológica; análise conceitual.

\section{Analysis of the Behavioral Variability Concept}

\begin{abstract}
The purpose of this paper is to analyze the concept of behavioral variability aiming at standardizing the different conceptions and uses of the term. We propose 1) a definition that resorts to the common characteristics underlying current variability concepts reported in the literature, and 2) the description and systematization of specific aspects of each one of those uses of the term by means of the following criteria: the content and structure of each concept, its empirical/ theoretical and molar/molecular levels of analysis, and types of measurement and codes under which they are specified. The present analysis is an initial attempt at obtaining a behavioral variability taxonomy.
\end{abstract}

Key words: behavioral variability; behavioral selection; learning; methodological analysis; conceptual analysis.

Mudança é a única constante.

(Lucretius, filósofo grego, há 2000 anos)

Em ciência, a variabilidade (ou variação de um fenômeno) pode ser analisada como um produto indesejável da pesquisa, um "ruído" decorrente da falta de controle experimental sobre variáveis desconhecidas ou mesmo de erro de mensuração. Essa variabilidade se entende que siga um padrão aleatório descrito por modelos probabilísticos, tais como o normal, o bi ou multinomial, entre outros. Entretanto, a variabilidade pode ser, em si mesma, um objeto de estudo, considerada como conseqüência de um conjunto de fatores de interesse temático. Sendo assim, fenômenos variáveis ou aparentemente caóticos vem sendo investigados em diferentes ciências (Bütz, 1995; Fivas, 1994; Thelen \& Smith, 1994), de forma que hoje buscam-se leis que governam a variabilidade da mesma forma como sempre se buscaram leis responsáveis pelos fenômenos mais repetitivos (Doll \& Freeman, 1986).

1 Este trabalho recebeu subvenção do $\mathrm{CNPq}$ (Brasil), através de Bolsa a Projeto Integrado de Pesquisa (processo no. 523.612/95-8) e parte da ajuda recebida pelo Grupo de Investigación (HUM-0287) do II Plan de Investigación de la Junta de Andalucía (Espanha). Os autores agradecem ao Dr. Armando Machado, Marcos Alexandre de Medeiros e Adriana Crunivel pela leitura crítica da primeira versão do manuscrito.

2 Endereço: Universidade de São Paulo, Departamento de Psicologia Experimental, Av. Prof. Mello Moraes, 1721, Cidade Universitária, CEP 05508-900, São Paulo, SP. E-mail: hunziker@usp.br.
No âmbito comportamental, a variabilidade - assim como a repetitividade - pode ser função de diferentes processos como, por exemplo, alguns estados fisiológicos alterados por drogas, lesões ou patologias diversas (Devenport, 1983). Além disso, nos organismos intactos, a variabilidade comportamental pode ser função de processos decorrentes da interação do organismo com seu meio ambiente. Como os demais fenômenos comportamentais, o da variabilidade pode ser considerado de duas perspectivas distintas: a do seu aparecimento inicial e a da sua manutenção. O aparecimento e a manutenção são mencionados de modo usual, embora às vezes confuso, como variação e seleção do comportamento, respectivamente, entendidos como processos complementares, necessários ambos à adaptação e sobrevivência dos indivíduos. Portanto, o termo variação tem um duplo significado, sendo às vezes utilizado para designar o fenômeno da variabilidade e em outros momentos utilizado para designar o processo de aparecimento de um novo comportamento. Dessa maneira, distinguindo-se fenômeno de processo, é possível se falar em processos de variação e seleção tanto da variabilidade como da repetitividade comportamental.

Apesar dessa complementaridade entre os processos de variação e seleção, a perspectiva behaviorista de análise do comportamento tem priorizado o estudo dos processos seletivos, sendo a sua ênfase dada nas relações de conseqüenciação que selecionam determinados padrões comportamentais, com pouca análise dos processos de variação des- 
ses comportamentos. Tal ordem de prioridade tem uma possível causa histórica, dado que tanto Thorndike (1932) quanto Skinner $(1966 ; 1981)$ enfatizaram os processos seletivos através da Lei do Efeito e do destaque da seleção pelas conseqüências, respectivamente (Arias, Fernández \& Benjumea, 1998; Richelle, 1986).

Atualmente, há uma vasta literatura relativa à variabilidade do comportamento produzida dentro de parâmetros definidos pelo arranjo experimental. Esses estudos seguem, basicamente, duas linhas gerais de investigação. A primeira, analisa a ocorrência da variabilidade como função de diferentes contingências de reforçamento que, contudo, não conseqüenciam a variação em si. Um experimento protótipo desse tipo de investigação foi realizado por Antonitis (1951) que submeteu ratos a diferentes contingências de reforçamento da resposta de tocar com o focinho uma barra horizontal dividida em espaços regulares de $1 \mathrm{~cm}$, totalizando 50 pontos que permitiam o registro da localização espacial da resposta. Os animais foram submetidos a diferentes contingências de reforçamento, na ordem que segue: nível operante (nenhuma conseqüenciação programada para a resposta de tocar a barra com o focinho), reforçamento contínuo (cada toque na barra, independentemente do ponto tocado, era seguido de reforçamento) e extinção (nenhum toque na barra era seguido de reforçamento). A variabilidade foi considerada relativa à diversidade dos pontos da barra tocados pelo sujeito, na escala dos 50 intervalos disponíveis. Os resultados revelaram que, em comparação ao nível operante, a variabilidade foi reduzida pelo reforçamento e aumentada pela suspensão do mesmo (extinção). Resultados como esses sugerem que a extinção pode induzir a variação do comportamento, mesmo não havendo reforçamento desse padrão comportamental (Eckerman \& Lanson, 1969; Lachter \& Corey, 1982; Schwartz, 1982). Na mesma linha, alguns autores obtiveram que o reforçamento intermitente também produz aumento de variabilidade comportamental, principalmente se envolver esquemas temporais tais como intervalo fixo ou variável (Boren, Moerschbaecher \& Whyte, 1978; Eckerman \& Vreeland, 1973; Ferraro \& Branch, 1968; Stebbins \& Lanson, 1962). Como a intermitência do reforçamento envolve, necessariamente, períodos de extinção (quando algumas das respostas da classe selecionada não são reforçadas), esses resultados inserem-se, aparentemente, na mesma linha de análise anteriormente citada. Vale destacar que nesses estudos, que utilizaram esquemas de reforçamento intermitente para investigar a variabilidade comportamental, a liberação do reforço não era contingente à variação do comportamento, sendo esta um "subproduto" da contingência em vigor. Portanto, diz-se que essa variabilidade - tanto decorrente da extinção quanto da intermitência do reforçamento - não é reforçada, mas sim induzida por condições ambientais.

A outra linha de investigação analisa se a liberação do reforço contingente à variação do comportamento produz um aumento da freqüência da resposta de variar. Num experimento protótipo desses estudos (Page \& Neuringer, 1985), pombos foram submetidos a reforçamento contingente à emissão de seqüências de quatro respostas de bicar dois discos localizados numa mesma parede da caixa experimental (discos direito-D e esquerdo-E). Nesse arranjo, o reforço foi liberado contingente à emissão da seqüência de respostas apenas se ela fosse diferente de "n" sequiências anteriores (procedimento lag- $n$ ). Essa diferença era avaliada comparativamente sobre a distribuição de respostas $\mathrm{D}$ ou $E$ dentro da seqüência (por exemplo, nas seqüências DDEE, DDEE, EDEE, DDEE, apenas a terceira difere de todas as anteriores). Sob essa contingência, se a sequiência fosse igual a uma das " $n$ " sequiências anteriores, ela não era seguida de reforçamento. De uma maneira geral, os estudos que utilizaram esse e outros procedimentos que envolviam reforçamento diferencial de padrões variáveis de comportamento - com ratos, pombos e humanos - vem indicando que há um controle operante dessa variabilidade na medida que 1) a variabilidade aumenta quando o reforçamento é contingente a ela, e decresce se esse reforçamento se dá independente dela (Machado 1989; Morgan \& Neuringer, 1990; Neuringer, 1986; 1992; Page \& Neuringer, 1985), e 2) pode ser colocada sob controle de estímulos (Neuringer, 1991; Page \& Neuringer, 1985). Esses estudos têm sugerido a ampliação da apregoada função seletiva do reforçamento, uma vez que o reforçamento pode não apenas selecionar respostas fixas ou repetitivas como também selecionar a sua variação (Neuringer, 1993; Page \& Neuringer,1985). Isso equivale a dizer que tais estudos colocam a seleção do comportamento variável - tanto quanto do repetitivo - num mesmo patamar de controle pelas conseqüências (Catania, 1998; Donahoe \& Palmer, 1994).

Apesar dessa aparente sistematicidade de resultados, o estudo da variabilidade do comportamento envolve questões teóricas/conceituais ainda a serem resolvidas. Um ponto crítico é a própria concepção geral sobre o fenômeno da variabilidade comportamental, a qual vem sendo expressa e medida de maneiras diversas. Conforme analisado por Barba (1996), os trabalhos sobre variabilidade comportamental podem ser agrupados segundo duas definições distintas - dispersão e eqüiprobabilidade - que redundam em diferentes mensurações e análises do comportamento. A noção de variabilidade como dispersão (por exemplo, Antonitis, 1951; Eckerman \& Lanson, 1969) pressupõe que o comportamento será tanto mais variável quanto mais se afastar de um valor central. O comportamento é, portanto, analisado sobre dados de variância, desvio padrão ou desvio médio, cujos valores apontam diretamente os graus de dispersão ou de variação. Enquanto eqüiprobabilidade, estudase a distribuição dos valores apresentados ao longo do universo de possibilidades considerando-se que quanto mais eqüitativa a distribuição dos eventos maior o grau de variabilidade (como em Denney, 1995; Ferraro \& Branch, 1968; Hunziker, Caramori, Silva \& Barba, 1998; Hunziker, Saldana \& Neuringer,1996; Machado 1989; 1992; 1993; Neuringer, 1986; 1991; 1992; Page \& Neuringer, 1985; Stokes, 1995). Geralmente se entende que essas relações ocorrem em graus, dentro de um continuum, sendo que eqüiprobabilidade representa o caso máximo de variabili- 
dade Dessa perspectiva, a variabilidade é analisada sobre índices estatísticos (U) que indicam incerteza, derivados da teoria da informação (Attenave, 1959).

A eqüiprobabilidade pode ser decorrente do fato de os eventos terem ocorrência independente entre si, o que, por sua vez, implica na impossibilidade de predição de um evento dada a ocorrência de outro. Portanto, nos casos em que os eventos são independentes, a sua eqüiprobabilidade de ocorrência é também indicadora da sua imprevisibilidade. Contudo, é possível que a distribuição eqüitativa dos eventos seja decorrente de um padrão altamente repetitivo, no qual todas as possibilidades de variação ocorrem de forma sistemática e repetitiva. Nesse caso, os eventos são altamente dependentes entre si e, se esse padrão puder ser detectado, são também altamente previsíveis. Portanto, os conceitos de eqüiprobabilidade e imprevisibilidade, embora sejam freqüentemente associados, devem ser analisados isoladamente.

Na prática, essas diferenças de conceituação geram, algumas vezes, conclusões distintas acerca dos mesmos dados. Por exemplo, no experimento de Antonitis (1951) podese supor dois comportamentos opostos: um rato que distribuísse suas respostas apenas nos extremos dessa barra (o que indicaria um alto índice de dispersão em relação ao ponto médio), e outro cujos toques se distribuíssem igualmente por todos os 50 intervalos (o que indicaria equiiprobabilidade de respostas). Adotando-se o conceito de dispersão, seria variável o comportamento do primeiro rato, enquanto que o conceito de eqüiprobabilidade apontaria resultado inverso, dando como variável o comportamento do segundo animal (Barba, 1996).

Outro problema conceitual, que foi apontado por Schwartz (1982), diz respeito à noção de reforçamento operante: se se considera que o reforçamento fortalece uma classe de respostas, qual é a propriedade objetiva que caracteriza as diferentes respostas como pertencentes à mesma classe de respostas variáveis? Ou seja, para se falar em variabilidade reforçada seria necessário explicitar a propriedade objetiva que define a classe de respostas reforçada. Contudo, sendo a variabilidade um conceito que depende da comparação entre classes de respostas, essa explicitação não pode se dar ao nível de uma propriedade inerente às respostas da classe reforçada.

Apontamos como problemático, também, a falta de consistência no uso da terminologia empregada. Por exemplo, num mesmo artigo os autores consideram a variabilidade ora como um operante (ou seja, um comportamento), ora como uma dimensão do comportamento operante, assim como o são força, duração, latência e topografia da resposta, entre outras (Neuringer, 1991; Page \& Neuringer,1985).

Algumas dessas questões conceituais vem sendo citadas na literatura de forma pontual (por exemplo, Machado, 1997) sem sofrer tratamento diferenciado dentro da área. Continua-se falando em variabilidade sem que o termo seja precisamente definido, o que, em ciência, pode representar uma fragilidade da área de investigação sendo, portanto, um problema que deve ser abordado. Nesse sentido, o estu- do da variabilidade comportamental pode se beneficiar de uma análise conceitual que explicite uma noção e critérios que permitam englobar sob o mesmo termo a diversidade de trabalhos existentes e outros que vierem a ser produzidos na área. O presente trabalho está sendo proposto com esse objetivo, ou seja, de fazer uma redefinição do termo através de uma análise conceitual da noção de variabilidade comportamental.

\section{Uma proposta de redefinição de variabilidade comportamental}

Entendemos que as diferentes definições formais e os usos do termo existentes abarcam aspectos do fenômeno, porém o fazem de forma incompleta e insuficientemente estruturadas entre si. Portanto, para se fazer uma redefinição sistematizadora consideramos necessárias duas tarefas complementares: 1) abstrair características que por serem comuns às várias definições ou usos do termo permitem reconhecê-las como tais e impliquem em uma noção geral desse fenômeno, e 2) descrever e sistematizar os aspectos específicos de cada uma dessas definições e usos, fazendo isso segundo alguns critérios que permitam entendê-las como casos particulares da noção geral.

De acordo com o uso cotidiano do termo, por variabilidade entendemos a diferença ou mudança entre elementos. Nesse sentido, a variabilidade comportamental pode ser caracterizada como diferença ou mudança entre comportamentos, aspecto esse coincidente com a característica comum que pode extrair-se de uma análise da literatura. Essa diferença ou mudança é um dos casos da comparação entre comportamentos que ocorrem em distintos momentos ou espaços, enquanto que um caso de igualdade de comportamentos comparados corresponderia à repetitividade ou falta de variabilidade comportamental. A existência de diferenças ou mudanças seria, portanto, o critério básico, necessário e suficiente para poder se falar de existência de variabilidade.

O critério de diferença torna-se mais claro ao se especificar ou definir a unidade e o universo comportamentais para a variabilidade considerados em cada referência a esse termo. Por unidade comportamental para a variabilidade entendemos cada uma das instâncias ou elementos comportamentais que são comparados, e cujas diferenças ou mudanças definem o fenômeno. Por universo comportamental de variabilidade entendemos o conjunto de unidades e comparações que definem o fenômeno. Contudo, o que em cada estudo constitui a unidade e o universo de variabilidade é algo convencional, no sentido que depende do ponto de vista ou decisão dos pesquisadores. Por exemplo, no contexto de um animal submetido a treinamento em esquema de reforçamento em intervalo fixo (FI), o universo de variabilidade poderia ser o conjunto de respostas que ocorrem em um intervalo, enquanto que a unidade seria cada uma das respostas emitidas nesse intervalo, comparadas entre si. Porém, esse mesmo conjunto de comportamentos também pode constituir a unidade de variabilida- 
de quando interessa analisar se há diferenças ou mudanças entre os diversos intervalos de uma sessão experimental, que constituiria em tal caso o universo. Outro exemplo, refere-se aos estudos que utilizam a análise de seqüências de respostas (como em Page \& Neuringer, 1985), onde o universo seria o conjunto de seqüências comportamentais apresentadas ao longo da sessão enquanto que a unidade seria cada seqüência emitida.

$\mathrm{Na}$ comparação que define a variabilidade, como em qualquer outra, uma unidade de comportamento é tomada sempre como referente em relação ao qual o outro comportamento é considerado diferente ou igual. Sendo sempre relativa a um determinado referente é, portanto, necessário o conhecimento desse referente para poder se falar de variabilidade do comportamento.

Dentro de um dado universo, as comparações entre unidades podem ser feitas de dois modos segundo o referente que seja utilizado: pode-se comparar cada uma das unidades com o resto (ou ao menos algumas delas com algumas das restantes), ou ainda tomar uma única unidade como referente e comparar o resto com ela. Este segundo caso se refere à variabilidade relativa a uma das unidades, como ocorre com a dispersão em relação a uma média, enquanto que no primeiro caso a variabilidade implicada é definida sem outorgar a primazia a um de seus valores e pondera de modo similar as diferentes unidades comparadas, como ocorre na medida de eqüiprobabilidade.

A distinção de unidade e universo comportamentais nos parece relevante também para responder à pergunta existente na literatura acerca da natureza da variabilidade reforçada: é um operante ou uma dimensão do comportamento operante? Do nosso ponto de vista, a variabilidade pode ser considerada como uma propriedade ou dimensão aplicável a um conjunto ou universo de comportamentos composto ao menos por dois elementos exigidos para se fazer uma comparação que defina tal fenômeno. Por isso, enquanto que dimensões como a força, duração, latência e topografia de estímulo ou da resposta são propriedades dos componentes de uma unidade comportamental, a propriedade de "ser variável" é pertinente a uma unidade em comparação com alguma outra, ou seja, pertinente a um universo ou conjunto de comportamentos comparados.

$\mathrm{O}$ fato de que a variabilidade pode ser considerada uma propriedade ou dimensão de um conjunto de comportamentos não impede que possa ser reforçada como padrão de comportamento desse conjunto. De fato, a literatura vem demonstrando que existem outras propriedades relacionais de conjuntos que são reforçados diferencialmente como, por exemplo, nos esquemas de reforçamento diferencial de baixas ou altas taxas de resposta (DRL e DRH, respectivamente), ou no reforçamento diferencial de IRTs (Ferster \& Skinner, 1957; Morse, 1966). Assim, pode-se responder ao problema levantado por Schwartz (1982) sobre a necessidade de se explicitar a propriedade objetiva que define o comportamento reforçado. Como temos dito, sendo variabilidade um conceito aplicável a um universo ou conjunto de comportamentos, a propriedade de cada comportamen- to para ser variável deve decorrer de uma comparação com um referente, sendo reforçada a diferença determinada em relação a ele. Assim, reforça-se a diferença que caracteriza aquela unidade de comportamento, da mesma maneira que são reforçados alguns IRTs ou determinadas taxas de resposta.

Concluímos que para se definir a variabilidade comportamental deve-se adotar como critério básico o requisito de diferença ou mudança entre unidades comportamentais. Falar de variabilidade é falar de diferenças entre certas unidades de algum universo determinado. Não há variabilidade a não ser entre unidades de algum universo, definidas ambas de modo convencional que resulte o mais relevante possível para entender o comportamento do sujeito em relação com seu ambiente. Por outro lado, necessitando-se sempre tomar uma unidade ou um conjunto delas como referente da comparação, variabilidade pode ser considerada relativa a esse referente, comum ou fixo para todas as comparações, ou em relação a unidades que mudam nas diferentes comparações. Em ambos os casos, a variabilidade acaba sendo uma propriedade de um universo de unidades comportamentais comparadas, e como tal pode ser reforçada e também induzida.

Considerando a definição anterior, é possível enumerar na noção de variabilidade uma série de aspectos particulares identificados em todo conceito de acordo com a descrição metodológica de Moreno, Martínez e Chacón (2000). Para isso, dois critérios básicos devem ser considerados: o conteúdo a que se refere o conceito e sua estrutura.

\section{Conteúdo da variabilidade}

Falar de conteúdo de variabilidade consiste em especificar o que é que varia. Por isso, o conteúdo de variabilidade é o conteúdo das unidades comportamentais de um universo dado que são comparadas. Coerente com o proposto por Skinner (1935), o comportamento seria definido como a relação organismo/ambiente, especificada em termos de qualquer das relações possíveis entre resposta $(\mathrm{R})$, ou unidade de tudo que o organismo faz, e estímulo (S), ou unidade de tudo que ocorre no meio ambiente. No geral, essas relações são expressas em termos da probabilidade de ocorrência de um dado evento em função da ocorrência de outro. ${ }^{3}$

Em consequiência, ainda que o conteúdo da variabilidade seja composto pelas unidades comportamentais, ela apenas pode ser considerada em termos das diferenças entre os componentes do comportamento, o que significa dizer al-

3 Cada resposta, ou estímulo, é um evento de ocorrência única. Para o estudo do comportamento convenciona-se agrupar esses eventos com todos aqueles que tenham ao menos uma propriedade comum, classificando-os como classes de respostas ou classes de estímulos, respectivamente. Cada classe é caracterizada por essa propriedade comum que define os eventos pertencentes a ela como ocorre, por exemplo, na classe de respostas de pressão à barra (Catania, 1998). Portanto, nesse texto, ao falarmos em respostas ou estímulos, entenda-se que estaremos sempre nos referindo a classes desses eventos. 
guma resposta e/ou algum estímulo, considerados uns e outros em termos de aspectos tais como topografia, duração, intensidade, freqüência, entre outros. Por exemplo, respostas de pressão à barra podem ocorrer com diferentes frequiências ao longo de uma sessão experimental, estímulos luminosos podem ser apresentados em diferentes momentos, o reforçamento pode ser estabelecido de diferentes maneiras na presença ou ausência desse estímulo: nesse caso, a análise do comportamento, enquanto estudo da variabilidade, se fará através das diferenças da freqüência de pressão à barra sob os diferentes esquemas de reforçamento ou com diferentes estímulos luminosos. ${ }^{4}$

Portanto, na medida que a variabilidade pode depender do universo e conteúdo dos comportamentos implicados, é conveniente que esses aspectos sejam sempre especificados ao se falar de variabilidade comportamental.

\section{Estrutura da variabilidade}

Falar de estrutura da variabilidade consiste em especificar sua composição. A idéia básica é que a definição de variabilidade como diferença ou mudança entre comportamentos tem uma estrutura relacional. Por isso, a estrutura da variabilidade é a estrutura das comparações, a qual depende da estrutura das unidades comportamentais de um universo determinado.

As unidades comportamentais podem ter diferentes estruturas, concretizadas em termos de número de variáveis de estímulo e de resposta, além da ordem de relações entre elas. O nível mais simples de comportamento implica uma relação de contingência de primeira ordem cujos componentes são apenas duas variáveis, como por exemplo, resposta e estímulo (convencionalmente chamada de relação de dois termos). Essa relação se define como covariações entre mudanças na resposta e mudanças ambientais no âmbito das diferenças entre a resposta emitida e as respostas anteriores. Um exemplo dessa relação encontra-se no trabalho de Antonitis (1951). Igualmente, nesse nível mais simples, as diferentes seqüências de respostas emitidas por um rato frente a duas barras seriam função desse comportamento ser, ou não, diferencialmente reforçado.

Um nível diferente de estrutura comportamental implica numa relação de segunda ordem, onde ao menos um dos seus componentes é uma relação de ordem inferior. É o caso, por exemplo, das relações de segunda ordem do tipo S-(R-S) (convencionalmente chamadas de relações de três termos) onde estímulos específicos (S) sinalizam diferentes contingências de reforçamento (relação R-S), contro-

4 Sob esse prisma, é curioso notar que embora o estímulo seja elemento componente da definição de comportamento, tanto quanto a resposta, o estudo da variabilidade comportamental tem priorizado apenas as diferenças observadas entre respostas. Contudo, um "velho" comportamento emitido numa nova situação (condição de generalização de estímulos) pode também ser considerado um novo comportamento e, nesse sentido, pode ser inserido dentro da área de investigação sobre variabilidade comportamental (Benjumea \& Arias, 1993). lando diferentes comportamentos. Um exemplo encontrase no trabalho de Page e Neuringer (1985) que mostra que a variabilidade e a repetitividade de sequiências de bicadas emitidas por pombos podem ser controladas por estímulos discriminativos, os quais sinalizam reforçamento contingente à variação ou à repetição da resposta, respectivamente.

Essa estrutura, definida em termos de número de variáveis e ordem das relações que variam é, ao menos teoricamente, ilimitada. Assim, complexidades maiores correspondem às relações de terceira ordem ou mais, convencionalmente classificadas como de quatro, cinco ou " $\mathrm{n}$ " termos. Além disso, em todos os níveis de relações anteriormente descritos pode-se ter ainda um aumento de complexidade em função do número de respostas e/ou estímulos componentes de cada relação. Por exemplo, no caso de uma relação de primeira ordem com dois termos, uma mesma classe de estímulo (tais como estímulos com determinada duração) pode alterar uma ou mais propriedades da classe de respostas à qual se relaciona (por exemplo, freqüência e/ou topografia da resposta). Da mesma maneira, diversas classes de estímulo podem se relacionar a uma ou diversas classes de resposta resultando, portanto, numa relação que poderia ser representada como $\left(\mathrm{S}_{1}, \ldots, \mathrm{S}_{\mathrm{n}}\right)-\left(\mathrm{R}_{1}, \ldots, \mathrm{R}_{\mathrm{n}}\right)$. Por sua vez, os universos podem conter dois ou mais comportamentos de qualquer das estruturas recém consideradas.

Uma estrutura com mais elementos de estímulo e resposta, e mais relações entre eles, implica em maiores possibilidades de variações. Assim, se a variabilidade pode depender da estrutura dos comportamentos e universo implicados, é conveniente que os estudos especifiquem sempre esses componentes do comportamento analisado.

\section{Modos de especificação da estrutura e conteúdo}

O conteúdo e a estrutura, enquanto critérios básicos do comportamento e sua variabilidade, constituem a base material desta. Porém, junto com sua base material, todo conceito apresenta um aspecto formal constituído pelos modos como conteúdos e estruturas são especificados pelos investigadores. Ocorre que cada especificação que se faça da variabilidade poderá afetar o modo como ela será considerada. Portanto, é necessário considerar essa especificação para esclarecer os aspectos que podem ser identificados na noção de variabilidade, condicionando as noções ou conceitos que se possam estabelecer sobre elas.

Essa especificação, ou expressão do comportamento tomado como unidade em estudo e de sua variabilidade, pode ser feita atendendo ao menos aos seguintes critérios: nível empírico ou teórico, análise molar ou molecular, tipo de medida ou valores que incluem, tipo de código com que se realiza a dita especificação.

Especificação empírica ou teórica - Dado um universo de comportamento definido basicamente por um determinado conteúdo e estrutura, a especificação dos comportamentos e sua variabilidade pode ser feita em dois níveis gerais. Algumas especificações permitem identificação da variabilidade em espaço e tempo concreto, o que se deno- 
mina especificação empírica. Diferentemente, as especificações chamadas teóricas descrevem o comportamento e sua variabilidade sem expressar diretamente essa concreção. As expressões de uma noção teórica não permitem, por si mesmas, a identificação empírica ainda que possam ser convertidas em noções empíricas.

Ambos aparecem nos diferentes estudos sobre a variabilidade comportamental. Um exemplo de especificação teórica é a afirmação de que a variabilidade do comportamento pode ser controlada por suas conseqüências, sendo exemplo da sua especificação empírica a demonstração experimental concreta de que animais apresentam seqüências de respostas mais variáveis se o reforçamento for contingente não apenas à emissão das sequiências como também à variabilidade das mesmas (Page \& Neuringer, 1985).

Esses dois níveis de especificação são relevantes e complementares, podendo, sem dúvida, interferir mutuamente um com o outro. Para investigar a possibilidade teórica de que a variabilidade comportamental poderia ser controlada por suas consequiências, Swartz (1982) estabeleceu um delineamento experimental onde pombos foram colocados frente a uma matriz quadrada composta por 25 lâmpadas (5X5), sendo exigido deles que movessem uma luz do canto superior esquerdo até o canto inferior direito: esse movimento da luz era produzido pela resposta de bicar um dentre dois discos (direito-D e esquerdo-E) que estavam constantemente disponíveis, de forma que bicadas em $\mathrm{D}$ movia a luz para baixo, enquanto que bicadas em E movia essa luz para a direita. Assim, para que a luz atingisse a posição requerida era necessária a emissão de sequiências de oito respostas de bicar, sendo exigência adicional que quatro respostas fossem dirigidas a cada disco. Essa foi a condição mínima para reforçamento, que produziu um relativo grau de variabilidade nas sequiências apresentadas. Porém, quando a essa exigência foi acrescentado o requisito de que a sequiência diferisse de algumas sequiências prévias para que o reforçamento ocorresse (procedimento lag-n), o comportamento se tornou mais repetitivo do que o obtido na ausência desse requisito. Tais resultados fundamentaram a conclusão de Swartz de que o reforçamento produzia repetição ao invés de variabilidade. Page e Neuringer (1985), por sua vez, argumentaram que o delineamento de Swartz continha um artefato que punia a variabilidade que se pretendia reforçar: a exigência de que a sequiência fosse composta por quatro respostas em cada disco limitava em 70 as sequiências passíveis de serem reforçadas, quando são 256 as combinações possíveis entre $\mathrm{D}$ e $\mathrm{E}$ numa seqüência de oito respostas. Assim, os pombos perdiam mais reforços por se comportarem de forma variável do que repetindo algumas poucas seqüências. O teste empírico dessa hipótese envolveu repetir o procedimento de Swartz, porém retirando-se a exigência de que metade das respostas fosse em cada disco. Os resultados de Page e Neuringer foram opostos aos de Swartz: os pombos apresentaram sequiências mais variáveis quando eram reforçados por isso do que quando o reforçamento independia da variabilidade desse comportamento. Devido a esses resultados, Page e Neuringer concluíram, contrariamente a Swartz, que a variabilidade do comportamento pode ser reforçada. Assim, uma mesma especificação teórica inicial sobre a variabilidade recebeu tratamentos empíricos distintos que produziram conclusões teóricas opostas sobre a natureza dessa variação.

Outro exemplo nessa área de estudo mostra uma relação diferente, onde duas especificações teóricas diversas sobre um mesmo tema geraram trabalhos experimentais com implicações distintas. Analisando-se os trabalhos que utilizam o procedimento de reforçamento de sequiências variáveis de respostas frente a duas barras ou discos (para ratos e pombos, respectivamente), identifica-se que esse procedimento implicitamente requer respostas de mudança de um manipulandum a outro para que as diferentes sequiências possam ocorrer. Esse fato recebe diferentes ênfases quanto à sua explicitação e relevância a nível teórico: alguns autores ignoram essas respostas de mudança e consideram que os dados existentes permitem afirmar que a variabilidade do comportamento é diretamente reforçada (Neuringer, 1993), enquanto outros afirmam que essa variabilidade é apenas um subproduto do reforçamento das respostas de mudança que freqüentemente não são analisadas nesses experimentos (Machado, 1997). Essas duas afirmações teóricas geram tratamentos empíricos diferenciados, produzindo experimentos com diferentes níveis de investigação sobre o processo de aprendizagem em curso e as possíveis implicações dos seus resultados. Assim, a premissa de que a variabilidade é diretamente reforçada vem sustentando estudos que investigam os efeitos de drogas, idade, sexo ou características genéticas dos sujeitos sobre essa variabilidade, sendo seus resultados analisados como tendo possíveis aplicações em nível educacional e terapêutico (Cohen, Neuringer \& Rhodes, 1990; Hunziker e cols., 1996; Neuringer, 1993; Neuringer \& Huntley, 1991). Por outro lado, a suposição de que a variabilidade, nesses experimentos ao menos, é apenas um subproduto do reforçamento direto da resposta de mudança tem gerado experimentos que investigam a natureza desse comportamento variável, ou seja, sobre o processo de aprendizagem que gera essa variabilidade (Barba, 1996; Machado, 1997). Esse detalhamento pode se expressar na análise da posição de cada resposta na sequiência emitida enquanto fator determinante da maior ou menor predição da resposta seguinte. Nesse estudos, os resultados são relacionados a questões básicas de análise do comportamento, como o controle de estímulos (Machado, 1997). Assim, duas especificações teóricas distintas geraram dois conjuntos de investigações empíricas diversas, um visando elucidar a própria estrutura do comportamento e seu processo de aprendizagem (Machado, 1997), enquanto outro buscou identificar variáveis que poderiam alterar um processo comportamental supostamente já conhecido (Neuringer e colaboradores).

Especificação molar e molecular - Dado um universo de comportamentos onde cada um é definido basicamente por um determinado conteúdo e estrutura, eles podem ser considerados de duas maneiras: detalhando seus diferentes componentes, o que supõe uma especificação em nível molecular, ou abstraindo os diferentes componentes dos comportamentos implicados e sua variabilidade, conside- 
rando-o de uma maneira global, o que caracteriza uma especificação em nível molar.

Por exemplo, no estudo da variabilidade comportamental, o conjunto dos trabalhos de Neuringer e seus associados considera a variabilidade fundamentalmente de forma molar, predominando a análise do grau de previsibilidade das sequiências emitidas ao longo do universo constituído por uma sessão experimental (por exemplo, Hunziker e cols., 1996; Morgan \& Neuringer, 1990; Neuringer, 1986; 1992; 1993). Por outro lado, além de fazer análises molares sobre o conjunto de comportamento ao longo das sessões, Machado $(1993 ; 1997)$ também vem realizando análises moleculares que permitem identificar o nível de dependência entre a localização de cada resposta dentro da sequiência e a sua probabilidade de emissão, como forma de identificar o que varia e qual o processo subjacente a essa variação.

Estes dois modos de especificação podem dar-se ao considerar variabilidade como desvio em relação a um valor central, bem como ao considerá-la em termos de eqüiprobabilidade. Em ambos os casos, a referência molar corresponde às noções e índices de variância e eqüiprobabilidade, respectivamente. A análise molecular é feita ao considerar cada diferença entre um dado valor e a referência central, no caso de dispersão, e a independência entre os diversos valores em eqüiprobabilidade.

A explicitação desse critério é relevante pois um processo de variabilidade que ocorre ao ser considerado em nível molar pode não ser necessariamente o mesmo que ocorre quando o seja em nível molecular. Retomando o exemplo do comportamento obtido sob esquema de reforçamento em FI, teríamos que, considerado o universo dos comportamentos de uma sessão ao nível molar, um animal bem treinado nesse esquema apresenta comportamento repetitivo: a inspeção visual de um registro cumulativo, obtido numa sessão típica de FI, revela um padrão recorrente onde a frequiência de respostas é baixa ou nula logo após o reforçamento, e aumenta visivelmente logo antes do final do intervalo; por outro lado, a análise molecular do comportamento dentro de cada intervalo nesse mesmo universo de uma sessão revela grande variabilidade comportamental, sugerindo controles distintos sobre o comportamento a cada momento do intervalo (Morse, 1966). Essa análise molecular permite um refinamento do estudo do controle temporal do comportamento, enquanto que a análise molar permite identificar padrões globais frente a essa contingência.

Tipos de medida especificada - Dado um universo de comportamentos, tanto cada comportamento como a comparação entre eles que permite falar de variabilidade podem apresentar-se em termos de valores ou especificações que impliquem distintos tipos de medidas.

Estas podem ser classificadas em níveis qualitativo, ordinal e quantitativo. Tanto um comportamento como sua comparação com algum outro são especificados com critérios qualitativos quando se indica apenas se dois valores desse comportamento, ou os de sua comparação com outros quaisquer, são iguais ou diferentes. Se dois valores do comportamento ou da comparação com outro forem dife- rentes, a sua distinção entre maior ou menor corresponderia ao modo ordinal de especificação. Por outro lado, se se indica quanto é a diferença entre dois valores, a especificação é quantitativa. Segundo mostram essas definições, cada tipo de valor se define como um detalhamento do anterior. $\mathrm{O}$ tipo ordinal detalha qual de dois valores qualitativamente diferentes é maior ou menor. O tipo quantitativo detalha quanto é maior ou menor um valor em respeito a outro. Essa progressiva inclusão permite, em sentido inverso, reduzir um determinado tipo ao anterior. Desse modo, os conceitos quantitativos (por exemplo, freqüência de uma resposta ou quantidade de variabilidade em termos de desvio em relação a um valor médio) podem ser considerados em termos ordinais (simplesmente tipo alta ou baixa frequiência, maior ou menor desvio) ou mesmo qualitativos (exemplo, especificando se ocorre ou não uma determinada freqüência, ou um determinado desvio).

Os diferentes tipos de medida podem dar-se num mesmo trabalho quando a variabilidade é considerada ao se compararem entre si todos os comportamentos de um universo. Por exemplo, no trabalho de Hunziker e cols. (1996), ratos foram reforçados por emitir sequiências de 4 respostas de pressão a duas barras - direita-D e esquerda-E - sendo o reforçamento contingente (ou não) à diferença entre as seqüências de respostas emitidas em $\mathrm{D}$ ou $\mathrm{E}$, dependendo da fase experimental. Na apresentação e discussão dos resultados desse estudo, o nível qualitativo de análise da variabilidade apareceu na comparação das configurações das seqüências (ou seja, da distribuição de D ou E nas quatro posições dentro da seqüência), o aspecto quantitativo deuse na análise da frequiência de emissão de cada uma dessas seqüências, enquanto que a análise ordinal apareceu na comparação entre maior ou menor diferença na frequiência de emissão das diferentes seqüências.

Tipos de códigos especificados - Dado um universo de comportamentos definidos cada um por um determinado conteúdo e estrutura, e especificado pelos modos possíveis, a variabilidade comportamental, como todo conceito em ciência, pode ser expressa através das seguintes formas: verbal, numérica simbólica e gráfica, segundo se utilize, respectivamente, códigos com semântica e sintaxe de alguma linguagem ordinária (por exemplo, inglês, espanhol, português), matemático formal (exemplo, números naturais, reais, racionais e complexos, expressões matriciais ou não matriciais das equações) ou icônico (histogramas, polígonos de freqüência, diagramas causais, etc). Assim como diferentes valores ou a análise molar/molecular podem conduzir às conclusões diferentes sobre variabilidade, o mesmo ocorre dependendo do código usado para representação do fenômeno em estudo. Por exemplo, determinadas representações gráficas podem tanto esconder como ressaltar de forma exagerada a variabilidade do comportamento, distorcendo a análise final desses dados. Igualmente, as conclusões que se pode alcançar através de representações gráficas ou análises estatístico/matemática podem ser conflitantes entre si sobre a existência ou não da variabilidade. 


\section{Conclusão}

A análise do termo variabilidade comportamental, baseada nos critérios aqui apresentados, permite um avanço nas questões conceituais inicialmente apontadas. Em primeiro lugar, a definição da variabilidade como diferença entre dois ou mais comportamentos permite que se aponte essa diferença como a propriedade comum que caracteriza conjuntos ou universos comportamentais. Assim como os intervalos entre respostas (IRTs) podem ser a propriedade comum que caracteriza algumas classes de respostas, sendo sensíveis à contingência operante, da mesma maneira podemos explicitar que a diferença entre comportamentos, independentemente do parâmetro mensurado em cada um (freqüência, topografia, força, localização espacial, etc), constitue a propriedade comum ao universo de comportamentos implicados como variáveis, sendo passível de ser modificada pelo reforçamento ou ser induzida.

Em segundo lugar, a conceituação aqui apresentada também permite uma melhor padronização o uso do termo. Aqui ficou explicitado que a variabilidade é entendida como um padrão descritivo de um conjunto ou universo de comportamentos, sendo que esse padrão consiste na diferença entre eles, especificada em graus de eqüiprobabilidade dentro do universo analisado, ou mesmo dado seu grau de dispersão em relação a um referente fixo. Por sua vez, foi proposto que esse universo de comportamentos variáveis pode ser considerado em termos de uma série de critérios identificáveis em toda noção ou conceito, os quais determinam a perspectiva que em cada caso se pode obter sobre variabilidade. Assim, pode não ser o mesmo falar de variabilidade em termos qualitativos ou quantitativos, em nível molecular ou molar, sobre um comportamento de nível de complexidade maior ou menor, com maior ênfase empírica ou teórica. O crítico é destacar que o que se estuda não é a variabilidade de forma unitária, mas sim variabilidade segundo os critérios considerados em cada caso. Cada referência à variabilidade pode ser diferente em função do critério ou aspecto analisado. Em conseqüência, consideramos que os trabalhos sobre o tema deveriam explicitar o critério que se está utilizando para definir a variabilidade.

Ao se considerar que os critérios que temos utilizado são dimensões de todo conceito, defendemos que na literatura podem-se encontrar referências explícitas ou usos implícitos deles, como mostram os exemplos apresentados nas seções anteriores. Desse modo, o conjunto de critérios que oferecemos pode ser utilizado como uma taxonomia dos usos do termo variabilidade encontrados na literatura, permitindo a organização do tema na medida em que cada uso desse termo poderia ser interpretado segundo alguns dos critérios.

É no seu uso que essa taxonomia será testada quanto à sua utilidade no avanço dos estudos sobre a variabilidade do comportamento. O que aqui propomos é apenas uma taxonomia inicial, a qual poderá ser modificada ou complementada em trabalhos futuros, dependendo da sua adequação ao tema que se propõe abarcar.

\section{Referências}

Antonitis, J.J. (1951). Response variability in the rat during conditioning, extinction, and reconditioning. Journal of Experimental Psychology, 42, 273-281.

Arias, M.F., Fernández, F. \& Benjumea, S. (1998). La ley del efecto y el origen de la conducta. Apuntes de Psicologia, 16 (3), 259-282.

Attenave, F. (1959). Applications of information theory to psychology: A summary of basic concepts, methods and results. New York: Holt-Dryden Book

Barba, L.S. (1996). Variabilidade comportamental aprendida. Dissertação de Mestrado, Universidade de São Paulo, São Paulo.

Benjumea, S. \& Arias, M.F. (1993). Pigeon' new behavior governed by multiple controlling stimuli. The Psychological Record, 43, 455-470.

Boren, J.J., Moerschbaecher, J.M. \& Whyte, A.A. (1978). Variability of response location on fixed-ratio and fixed-interval schedules of reinforcement. Journal of the Experimental Analysis of Behavior, 30, 63-67.

Bütz, M.R. (1995). Chaos theory, philosophically old, scientifically new. Counseling and Values, 39 (2), 84-34.

Catania, A.C. (1998). Learning. Englewood Cliffs, N.J.:PrenticeHall, Inc. (4a. edição)

Cohen, L., Neuringer, A. \& Rhodes, D. (1990). Effects of ethanol on reinforced variations and repetitions by rats under a multiple schedule. Journal of the Experimental Analysis of Behavior, 54, 1-12.

Denney, J. (1995). Stimulus control of behavioral variability. Tese de conclusão de curso, Reed College, Portland (USA).

Devenport, L.D. (1983). Spontaneous behavior: Inferences from neurosciences. Em R. Molgren (Org.), Animal cognition and behavior (pp. 83-125). Amsterdam: North-Holland.

Doll, J.D. \& Freeman, D.L. (1986). Randomly exact methods. Science, 234, 1356-1360.

Donahoe, J.W. \& Palmer, D.C. (1994). Learning and complex behavior. Boston (MA): Allyn and Bacon.

Eckerman, D.A. \& Lanson, R.N. (1969). Variability of response location for pigeons responding under continuous reinforcement, intermittent reinforcement, and extinction. Journal of the Experimental Analysis of Behavior, 12, 73-80.

Eckerman, D.A. \& Vreeland, R. (1973). Response variability for humans receiving continuous, intermittent, or no positive experimenter feedback. Bulletin of the Psychonomic Society, 2, 297-299.

Ferraro, D.P. \& Branch, K.H. (1968). Variability of response location during regular and partial reinforcement. Psychological Reports, 23, 1023-1031.

Ferster, C.B. \& Skinner, B.F. (1957). Schedules of reinforcement. New York: Appleton-Century-Crofts.

Fivas, R. (1994). The nature of order in complex systems. Systems Research, 11 (3), 43-65.

Hunziker, M.H.L., Caramori, F.C., Silva, A.P. \& Barba, L. (1998). Efeitos da história de reforçamento na variabilidade comportamental. Psicologia: Teoria e Pesquisa, 14 (2), 149-159. 
Hunziker, M.H.L., Saldana, L. \& Neuringer, A. (1996). Behavioral variability in SHR and WKY rats as a function of rearing environment and reinforcement contingency. Journal of the Experimental Analysis of Behavior, 65 (1), 129-144.

Lachter, G.D. \& Corey, J.R. (1982). Variability of the duration of an operant. Behavior Analysis Letters, 2, 97-102.

Machado, A. (1989). Operant conditioning of behavioral variability using percentile reinforcement schedule. Journal of the Experimental Analysis of Behavior, 52, 155-166.

Machado, A. (1992). Behavioral variability and frequency-dependent selection. Journal of the Experimental Analysis of Behavior, 58, 241-263.

Machado, A. (1993). Learning variable and stereotypical sequences of responses: some data and a new model. Behavioral Processes, 30, 103-130.

Machado, A. (1997). Increasing the variability of response sequences in pigeons by adjusting the frequency of switching between two keys. Journal of Experimental Analysis of Behavior, 68, 1-25..

Moreno, R., Martínez, R. \& Chacón, S. (2000). Fundamentos de metodología en psicología y ciencias afines. Madrid: Pirámide.

Morgan, L. \& Neuringer, A. (1990). Behavioral variability as a function of response topography and reinforcement contingency. Animal Learning and Behavior, 18, 257-263.

Morse, W.H. (1966). Intermittent reinforcement. Em W.K. Honig (Org.), Operant behavior: Areas of research and application (pp 52-108). New York: Appleton-Century-Crofts.

Neuringer, A. (1986), Can people behave "randomly"?: The role of feedback. Journal of Experimental Psychology: General, $115,62-75$.

Neuringer, A. (1991). Operant variability and repetition as functions of interresponse time. Journal of experimental Psychology: Animal Behavior Processes, 17, 3-12.
Neuringer, A. (1992). Choosing to vary and repeat. Psychological Science, 3, 246-250.

Neuringer, A. (1993). Reinforced variation and selection. Animal Learning and Behavior, 21 (2), 83-91.

Neuringer, A. \& Huntley, R.W. (1991). Reinforced variability in rats: Effects of gender, age and contingency. Physiology and Behavior, 51, 145-149.

Page, S. \& Neuringer, A. (1985). Variability is an operant. Journal of Experimental Psychology: Animal behavior Processes, 11, 429-452.

Richelle, M. (1986). Variation and selection: The evolutionary analogy in Skinner's theory. Em S. Modgil e C. Modgil (Org.), B.F. Skinner: Consensus and controversy (pp 127-137). New York: Falmer.

Schwartz, B. (1982). Failure to produce response variability with reinforcement. Journal of the Experimental Analysis of Behavior, 37, 171-181.

Skinner, B.F. (1935). The generic nature of the concepts of stimulus and response. Journal of General Psychology, 12, 40-65.

Skinner, B.F. (1966). The ontogeny and phylogeny of behavior. Science, 153, 1203-1213.

Skinner, B.F. (1981). Selection by consequences. Science, 213, 501-504.

Stebbins, W.C. \& Lanson, R.N. (1962). Response latency as a function of reinforcement schedule. Journal of the Experimental Analysis of Behavior, 5, 299-304.

Stokes, P. (1995). Learned variability. Animal Learning and Behavior, 23, 164-176.

Thelen, E. \& Smith, L.B. (1994). A dynamic: Systems approach to the development of cognition and action. Cambridge: MIT Press.

Thorndike, E.L. (1932). The fundamentals of learning. New York: Columbia University Press. 